\title{
Short-Term Apixaban for Documented Left Atrial Appendage Thrombus in High-Risk Atrial Fibrillation Patients Undergoing Left Atrial Appendage Occlusion
}

\author{
Wern Yew Ding ${ }^{1}$ Gregory Y.H. Lip ${ }^{1,20}$ Timothy Fairbairn $^{3}$ Sukumaran Binukrishnan ${ }^{3}$ \\ Afshin Khalatbari ${ }^{3}$ Periaswamy Velavan ${ }^{3}$ Dhiraj Gupta ${ }^{1}$
}

${ }^{1}$ Liverpool Centre for Cardiovascular Science, University of Liverpool and Liverpool Heart and Chest Hospital, Liverpool, United Kingdom

2 Department of Clinical Medicine, Aalborg Thrombosis Research Unit, Aalborg University, Aalborg, Denmark

${ }^{3}$ Department of Cardiology, Liverpool Heart and Chest Hospital, Liverpool, United Kingdom

TH Open 2020;4:e351-e353.

Stroke prevention with left atrial appendage (LAA) occlusion is an important alternative treatment for high-risk atrial fibrillation (AF) patients with contraindications to oral anticoagulation $(\mathrm{OAC})^{1,2}{ }^{1,2}$ The presence of LAA thrombus detected on preprocedural imaging usually precludes implantation of the endocardial device because of a prohibitively high periprocedural embolic risk. ${ }^{3}$ Although successful resolution of LAA thrombus with OAC has been described, all previous reports are in patients without contraindications to $\mathrm{OAC}^{4-6}$ Furthermore, long-term data in these patients are lacking. We hypothesized that short-term OAC with apixaban could safely facilitate resolution of LAA thrombi even in a high-risk population, thereby allowing safe LAA occluder device implantation, with good outcomes over the long term.

This was a retrospective cohort study of 83 patients who underwent work-up for LAA occlusion at our center between January 2015 and December 2017. LAA thrombi were documented in $11(13.3 \%$ ) patients (mean age: 76.9 [ \pm 6.9 ] years; 7 males) on preprocedural imaging (9 on transesophageal echocardiography and 2 on cardiac computed tomographic angiography) (-Table 1). All patients with LAA thrombi had permanent $\mathrm{AF}$, with median $\mathrm{CHA}_{2} \mathrm{DS}_{2}$-VASc and HAS-BLED scores of 4 (interquartile range [IQR]: 3-5) and 2 (IQR: 2-3), respectively. Contraindications to lifelong OAC in this cohort were prior intracranial hemorrhage in six patients (two on warfarin, one on dabigatran, one on heparin, and two while not on anticoagulation), prior major gastrointestinal hemorrhage requiring emergency hospital admission and blood transfusion in three patients (two on warfarin and one while
Address for correspondence Dhiraj Gupta, MD, Liverpool Centre for Cardiovascular Science, Liverpool Heart and Chest Hospital, Thomas Drive, Liverpool, L14 3PE, United Kingdom (e-mail: Dhiraj.Gupta@lhch.nhs.uk). not on anticoagulation), severe unexplained anemia on dabigatran in one patient, and failed OAC in one patient. At the time of imaging, none of the patients were receiving OAC, and four (36.4\%) patients were on a single antiplatelet agent. There were no significant differences between patients who had documented LAA thrombus compared with those without in terms of age ( $p=0.80$ ), sex category ( $p=0.74)$, AF type $(p=0.11)$, comorbidities (hypertension $[p=0.50]$, diabetes mellitus $[p=0.31]$, and prior stroke or transient ischemic attack [ $p=0.47])$, and $\mathrm{CHA}_{2} \mathrm{DS}_{2}$-VASc score $(p=0.69)$.

The potential risks of surgical thrombectomy and ligation were felt to outweigh the benefits. Hence, following detection of LAA thrombi, off-label dose-adjusted apixaban ( $2.5 \mathrm{mg}$ twice daily for three patients and $5 \mathrm{mg}$ twice daily for eight patients) was prescribed for each patient. The choice of anticoagulation was based on evidence of potential favorable characteristics of apixaban compared with other OAC in terms of efficacy and safety profile. ${ }^{7}$ Repeat imaging in the form of transesophageal echocardiography, or cardiac computed tomographic angiography was scheduled at 6- to 8-week intervals. Complete resolution of LAA thrombus was observed in 10 (90.9\%) patients after apixaban treatment for a median of 94 days (IQR: 44-126 days). During treatment with apixaban, one patient (who received a dose of $5 \mathrm{mg}$ twice daily) had a severe gastrointestinal bleed requiring blood transfusion, and one patient suffered an ischemic stroke with subsequent full recovery. There was no significant difference in the risk of bleeding according to apixaban dosage $(p=0.73)$. One patient (patient $8,-$ Table 1$)$ had persistent LAA thrombus on repeated imaging, and a received

July 14,2020

accepted

September 7, 2020
DOI https://doi.org/ 10.1055/s-0040-1718585. ISSN 2512-9465.
๑) 2020 Georg Thieme Verlag KG
Stuttgart · New York

License terms

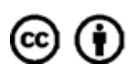


Table 1 Characteristics of patients with left atrial appendage thrombus

\begin{tabular}{|c|c|}
\hline Patients & Characteristics \\
\hline Patient 1 & $\begin{array}{l}\text { Age: } 82 \text { y; eGFR: } 42 \text {; } \mathrm{CHA}_{2} \mathrm{DS}_{2} \text {-VASc: 3; HAS-BLED: 2; recurrent Gl bleed on warfarin; OGD revealed gastric } \\
\text { angiodysplasia; CKD; COPD; angina }\end{array}$ \\
\hline Patient 2 & $\begin{array}{l}\text { Age: } 76 \text { y; eGFR: 74; } \mathrm{CHA}_{2} \mathrm{DS}_{2} \text {-VASc: 5; HAS-BLED: 2; recurrent GI bleed on warfarin; no source identified on OGD and } \\
\text { colonoscopy }\end{array}$ \\
\hline Patient 3 & $\begin{array}{l}\text { Age: } 75 \text { y; eGFR: } 90 ; \mathrm{CHA}_{2} \mathrm{DS}_{2} \text {-VASc: } 4 \text {; HAS-BLED: 3; massive GI bleed while off anticoagulation; OGD identified variceal } \\
\text { source, which was treated with banding; nonalcoholic liver cirrhosis; old inferior MI; mild LV dysfunction; hypothyroidism }\end{array}$ \\
\hline Patient 4 & $\begin{array}{l}\text { Age: } 78 \text { y; eGFR: } 53 \text {; } \mathrm{CHA}_{2} \mathrm{DS}_{2} \text {-VASc: 5; HAS-BLED: 2; ICH on heparin for newly diagnosed atrial fibrillation; prior ischemic } \\
\text { stroke; CKD; HTN; hypothyroidism }\end{array}$ \\
\hline Patient 5 & $\begin{array}{l}\text { Age: } 81 \text { y; eGFR: 64; } \mathrm{CHA}_{2} \mathrm{DS}_{2} \text {-VASc: 3; HAS-BLED: 3; ICH on warfarin (subtherapeutic international normalized ratio); } \\
\text { meningioma; epilepsy; HTN; prior pneumothorax }\end{array}$ \\
\hline Patient 6 & $\begin{array}{l}\text { Age: } 80 \text { y; eGFR: } 48 ; \mathrm{CHA}_{2} \mathrm{DS}_{2} \text {-VASc: 4; HAS-BLED: 3; ICH on dabigatran; CKD; epilepsy; uncontrolled HTN; treated breast } \\
\text { cancer }\end{array}$ \\
\hline Patient 7 & $\begin{array}{l}\text { Age: } 68 \text { y; eGFR: 47; } \mathrm{CHA}_{2} \mathrm{DS}_{2} \text {-VASc: 6; HAS-BLED: 2; ICH while off anticoagulation; cerebral amyloid angiopathy; prior } \\
\text { stroke; old MI; severe LV dysfunction; CKD; HTN }\end{array}$ \\
\hline Patient 8 & Age: 88 y; eGFR: 67; $\mathrm{CHA}_{2} \mathrm{DS}_{2}$-VASc: 3; HAS-BLED: 2; large ICH on warfarin; HTN \\
\hline Patient 9 & $\begin{array}{l}\text { Age: } 67 \text { y; eGFR: 75; } \mathrm{CHA}_{2} \mathrm{DS}_{2} \text {-VASc: 5; HAS-BLED: 3; ICH while off anticoagulation; recurrent stroke and transient } \\
\text { ischemic attack; prior pulmonary embolism and deep venous thrombosis; seizures; left ventricular hypertrophy }\end{array}$ \\
\hline Patient 10 & $\begin{array}{l}\text { Age: } 83 \text { y; eGFR: } 44 ; \mathrm{CHA}_{2} \mathrm{DS}_{2} \text {-VASc: 4; HAS-BLED: 2; severe anemia of unknown origin while on dabigatran; CKD; left } \\
\text { bundle branch block; HTN }\end{array}$ \\
\hline Patient 11 & $\begin{array}{l}\text { Age: } 68 \text { y; eGFR: 67; } \mathrm{CHA}_{2} \mathrm{DS}_{2} \text {-VASc: 5; HAS-BLED: 3; ischemic stroke on apixaban; cerebral amyloid angiopathy; prior } \\
\text { embolic stroke; polycythemia; COPD; alcohol excess; HTN }\end{array}$ \\
\hline
\end{tabular}

Abbreviations: CKD, chronic kidney disease; COPD, chronic obstructive pulmonary disease; eGFR, estimated glomerular filtration rate; GI, gastrointestinal; HTN, hypertension; ICH, intracranial hemorrhage; LV, left ventricular; MI, myocardial infarction; OGD, esophagogastroduodenoscopy.

patient-centered decision was ultimately taken for continued apixaban therapy despite prior major intracranial bleed while on warfarin. In this patient, no bleeding complications were observed over a follow-up period of 25 weeks.

The 10 patients with thrombus resolution underwent successful LAA occlusion with no periprocedural complications. Apixaban was replaced by dual-antiplatelet therapy with aspirin and clopidogrel for 6 weeks postprocedure followed by single (or no) antiplatelet therapy. The decision to discontinue long-term antiplatelet therapy in three patients was based on an individualized approach after accounting for their high bleeding risk. All patients underwent cardiac imaging with transesophageal echocardiography or cardiac computed tomographic angiography at 6 to 8 weeks postprocedure; no device-related thrombus was observed in any patient. Across a median follow-up of 2.5 years (IQR: 0.6-3.3 years), one patient suffered a transient ischemic attack and another had an episode of severe epistaxis despite not being on antiplatelet or OAC therapy at the time. During this same period, four (40\%) patients died.

Given the low number of patients, our study was underpowered and should therefore be interpreted with caution. Furthermore, the incidence of LAA thrombus in our study may not reflect those of the general AF population as many of our high-risk patients were not on anticoagulation therapy at baseline. It is also worth noting that we adopted a strategy of minimizing the exposure to OAC in this high-risk cohort by performing LAA occlusion without delay in patients with a confirmed resolution of LAA thrombi.
Our experience suggests that short-term treatment with apixaban may be effective and safe for aiding the resolution of documented LAA thrombus in high-risk patients who are ineligible for lifelong OAC therapy. This strategy allows the LAA occlusion procedure to be undertaken safely, thereby potentially reducing the long-term risk of thromboembolism.

\section{Conflict of Interest}

G.Y.H.L. is a consultant for Bayer/Janssen, BMS/Pfizer, Medtronic, Boehringer Ingelheim, Novartis, Verseon, and Daiichi-Sankyo and speaker for Bayer, BMS/Pfizer, Medtronic, Boehringer Ingelheim, and Daiichi-Sankyo. No fee is directly received personally. D.G. is a speaker for Bayer, BMS/Pfizer, Boehringer Ingelheim, Daiichi-Sankyo, Medtronic, Biosense Webster, and Boston Scientific and proctor for Abbott. Research grants from Medtronic, Biosense Webster, and Boston Scientific were received. The funders had no role in the submitted paper. D.G. reports personal fee from Abbott. The other authors report no conflict of interests.

\section{References}

1 Freeman JV, Varosy P, Price MJ, et al. The NCDR Left Atrial Appendage Occlusion Registry. J Am Coll Cardiol 2020;75(13): 1503-1518

2 Ding WY, Mandrola J, Gupta D. Left atrial appendage occlusion: past, present and future. Thromb Haemost 2020(e-pub ahead of print).. Doi: 10.1055/s-0040-1714654 
3 De Backer O, Arnous S, Ihlemann N, et al. Percutaneous left atrial appendage occlusion for stroke prevention in atrial fibrillation: an update. Open Hear 2014;1(01):e000020

4 Fleddermann A, Eckert R, Muskala P, Hayes C, Magalski A, Main ML. Efficacy of direct acting oral anticoagulant drugs in treatment of left atrial appendage thrombus in patients with atrial fibrillation. Am J Cardiol 2019;123(01):57-62

5 Piotrowski R, Zaborska B, Pilichowska-Paszkiet E, Sikora-Frąc M, Baran J, Kułakowski P. RIVAroxaban TWICE daily for lysis of thrombus in the left atrial appendage in patients with nonvalvular atrial fibrillation: the RIVA-TWICE study. Arch Med Sci 2019;16(02):289-296
6 Lip GYH, Hammerstingl C, Marin F, et al; X-TRA study and CLOTAF registry investigators. Left atrial thrombus resolution in atrial fibrillation or flutter: Results of a prospective study with rivaroxaban (X-TRA) and a retrospective observational registry providing baseline data (CLOT-AF). Am Heart J 2016; 178:126-134

7 Lip GYH, Mitchell SA, Liu X, et al. Relative efficacy and safety of non-Vitamin K oral anticoagulants for non-valvular atrial fibrillation: Network meta-analysis comparing apixaban, dabigatran, rivaroxaban and edoxaban in three patient subgroups. Int J Cardiol 2016;204:88-94 